\title{
OPEN Microdrilled tapers to enhance optical fiber lasers for sensing
}

\author{
R. A. Perez-Herrera ${ }^{1,2 \bowtie}$, M. Bravo ${ }^{1,2}$, P. Roldan-Varona ${ }^{3,4,5}$, D. Leandro ${ }^{1,2}$, L. Rodriguez-Cobo ${ }^{4}$, \\ J. M. Lopez-Higuera ${ }^{3,4,5}$ \& M. Lopez-Amo ${ }^{1,2}$
}

In this work, an experimental analysis of the performance of different types of quasi-randomly distributed reflectors inscribed into a single-mode fiber as a sensing mirror is presented. These artificially-controlled backscattering fiber reflectors are used in short linear cavity fiber lasers. In particular, laser emission and sensor application features are analyzed when employing optical tapered fibers, micro-drilled optical fibers and $50 \mu \mathrm{m}$-waist or $100 \mu \mathrm{m}$-waist micro-drilled tapered fibers (MDTF). Single-wavelength laser with an output power level of about $8.2 \mathrm{dBm}$ and an optical signal-to-noise ratio of $45 \mathrm{~dB}$ were measured when employing a $50 \mu \mathrm{m}$-waist micro-drilled tapered optical fiber. The achieved temperature sensitivities were similar to those of FBGs; however, the strain sensitivity improved more than one order of magnitude in comparison with FBG sensors, attaining slope sensitivities as good as $18.1 \mathrm{pm} / \mu \varepsilon$ when using a $50 \mu \mathrm{m}$-waist MDTF as distributed reflector.

Today, fiber Bragg grating (FBG) based sensors are well-stablished transducers for a great number of sensing applications ${ }^{1}$. They are especially well suited for strain and temperature measurements. Thus, they are extensively used for monitoring civil works or in sensing platforms including a large number of this type of sensors ${ }^{2}$. Their main advantage is that the measured parameter is coded in a wavelength shift, which is immune to opticalpower fluctuations. Moreover, because they are wavelength-selective reflectors, they can be employed as a part of an optical laser cavity. Due to this, since 1993 FBG sensing systems based on optical fiber lasers have been developed $^{3}$. These lasing sensors have demonstrated a high sensitivity and a high optical signal-to-noise ratio $(\mathrm{OSNR})^{4}$. Typically, FBG sensing systems offer a temperature sensitivity of $11 \mathrm{pm} /{ }^{\circ} \mathrm{C}$ and a strain sensitivity of $1.2 \mathrm{pm} / \mu \varepsilon^{5,6}$. These sensitivities have been enough to establish this type of sensors as a flagship in sensing along more than two decades.

On the other hand, micro-drilling techniques, such as the employed in this work, have shown a high potential recently, not only in FBGs writing ${ }^{7}$, but also to develop enhanced optical fiber lasers and more sensitive sensing structures. Femtosecond (fs) laser can offer strong refractive index variations without the requirement of a photosensitivity enhancement technique ${ }^{8}$. This is achieved by using ultrashort pulses and extreme high instantaneous power. The fs laser can also induce non-linear multi-photon absorption of materials to locally change the refractive index of the core of a SMF. Thus, backscattering reflectors can be fabricated inside the core of an optical fiber. A number of laser generation applications of these artificially controlled backscattering fiber reflectors (ACBFRs) have been previously demonstrated. This type of distributed reflectors can be inscribed along different types of optical fiber. Previous works experimentally demonstrated their properties for distributed short-linear-cavity fiber lasers ${ }^{9}$ or random fiber laser generation ${ }^{10}$ assisted by ACBFRs inscribed along a singlemode fiber. On the other hand, it has also been experimentally demonstrated that multi-wavelength emission with single-longitudinal mode (SLM) behavior can be obtained when these distributed reflectors are inscribed not in a standard SMF but along a highly-doped erbium fiber ${ }^{11}$. It this case, ACBFRs act not only as distributed reflectors but also as saturable absorbers, obtaining up to eight simultaneous SLM emission lines.

In this work, we have developed quasi-randomly distributed reflectors inscribed into a standard single-mode or tapered optical fiber with different waist dimensions as a sensing mirror. Their performances in fiber laser configurations are experimentally evaluated. By using this type of mirrors, it is also possible to develop shortcavity fiber optic laser sensors which offer higher strain sensitivity than regular FBG transducers. In particular, the laser generation and sensor application features when optical tapered fibers (TF), micro-drilled optical fibers (MDF) or two different micro-drilled tapered fibers (MDTF) are presented.

${ }^{1}$ Department of Electrical Electronic and Communication Engineering, Public University of Navarra, 31006 Pamplona, Spain. ${ }^{2}$ Institute of Smart Cities (ISC), Public University of Navarra, 31006 Pamplona, Spain. ${ }^{3}$ Photonics Engineering Group, University of Cantabria, 39005 Santander, Spain. ${ }^{4} \mathrm{CIBER}$-Bbn, Instituto de Salud Carlos III, 28029 Madrid, Spain. ${ }^{5}$ Instituto de Investigacion Sanitaria Valdecilla (IDIVAL), 39005 Cantabria, Spain. ${ }^{\square}$ email: rosa.perez@unavarra.es 


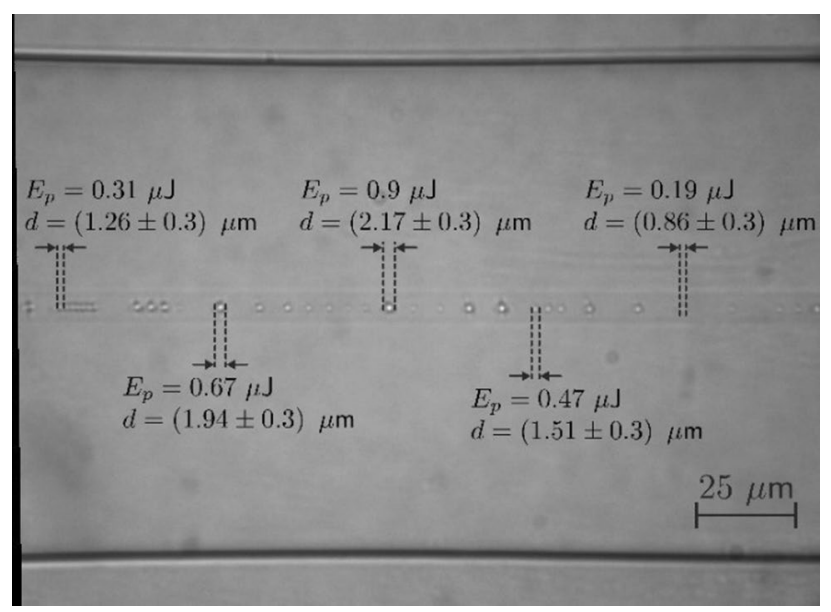

Figure 1. Microscope image of the quasi-randomly distributed reflector along the SMF.

\section{Working principle: inscription process}

In this experimental study, three different distributed reflectors based on tapered optical fibers or/and microdrilled optical fibers were employed. A standard transversal inscription setup, like the one employed in ${ }^{9}$, was selected. The distributed reflectors have been manufactured using a femtosecond commercial Fiber Laser Chirped Pulse Amplifier (FLCPA) from CALMAR lasers, operating at $1030 \mathrm{~nm}$ wavelength, with 370 fs of pulse duration, and a variable pulse repetition (PRR) rate available up to $120 \mathrm{kHz}$. Femtosecond laser processing provides inhomogeneity enhancement of the refractive index of the fiber ${ }^{12}$, increasing the distributed scattering. The fiber, located over a nano-resolution Aerotech stage motor, is placed on a slide and covered with a coverslip. Between them, an index-matching oil is deposited to limit fiber-induced aberrations ${ }^{13}$. Then, the laser pulses are tightly focused through a $100 \times / \mathrm{NA}=0.5$ objective lens from Mitutoyo Corporation. Regarding the inscription parameters, it is worth noting that pseudo-randomly varying pulse energies between 0.19 and $0.9 \mu \mathrm{J}$ have been used (as can be seen in Fig. 1). Likewise, the period $(\Lambda)$ between each laser spot is pseudo-randomly modified between 1 and $10 \mu \mathrm{m}$.

Micro-drilled standard single-mode optical fiber (MDF). In the first study, a micro-drilled standard single-mode optical fiber (MDF), with a $125 \mu \mathrm{m}$ diameter was used. Figure 1 depicts a microscope image of the quasi-randomly distributed reflector along the fiber, showing the dimensions of some modifications induced along the SMF. As it may be seen in this figure, the random location of the induced changes was achieved by randomly modulating the pulse repetition rate at every pulse (which corresponds to $100 \mathrm{~ms}$ approximately). Consequently, a quasi-random inscribed structure with several micro-drilled points was attained. However, the repetition rate of the femtosecond laser could not be simultaneously modulated at the same rate as the pulseinscription speed. Thus, direct random inscription was not possible, but quasi-randomly spaced spots was.

Micro-drilled tapered optical fiber (MDTF): $100 \mu \mathrm{m}$-waist tapered fiber. Secondly, two different micro-drilled tapered optical fibers (MDTF) were developed. Both of them were fabricated from standard single-mode fiber (SMF). The fabrication of the tapered fibers was carried out by means of a Taper Manufacturing Station TMS-01-0400 (3SAE) (NorthLab Photonics, Sweden), which allows manufacturing tapered fibers with arbitrary shapes, low losses, and excellent repeatability, as has been previously reported ${ }^{14}$. The first inscription process was carried out from a $100 \mu \mathrm{m}$-waist tapered fiber. The transitions between the original optical fiber and the uniform waist of the taper were of around $5 \mathrm{~mm}$ each, for a total taper length of about $30 \mathrm{~mm}$, as can be seen in Fig. 2.

Micro-drilled tapered optical fiber (MDTF): $50 \mu \mathrm{M}$-waist tapered fiber. Finally, for the second MDTF, a $50 \mu \mathrm{m}$-waist was employed. A schematic illustration of this micro-drilled $50 \mu \mathrm{m}$-waist tapered optical fiber is depicted in Fig. 2. In this case, the transitions between the original SMF and the uniform $50 \mu \mathrm{m}$-waist were also of $5 \mathrm{~mm}$ each and a total taper length of $30 \mathrm{~mm}$ was also carried out.

\section{Experimental setup}

Figure 3 shows a schematic diagram of the experimental setup used to evaluate the laser generation and sensor properties when using the distributed reflectors within a short-linear-cavity fiber acting as a mirror. In this figure, the three types of reflectors employed can be seen: (a) micro-drilled optical fiber (MDF), (b) un-drilled tapered optical fibers (TF), and (c) micro-drilled tapered fibers (MDTF).

A 980/1550 nm wavelength-division multiplexer (WDM) injects the pump laser centered at $976 \mathrm{~nm}$ into the linear-cavity fiber laser. The gain medium, located at the common port of the WDM, consists of $4 \mathrm{~m}$ of erbium-doped fiber (EDF) ${ }^{9}$. The EDF was the I25 (980/125) (Fibercore Inc.), suitable for C amplifiers with a core 


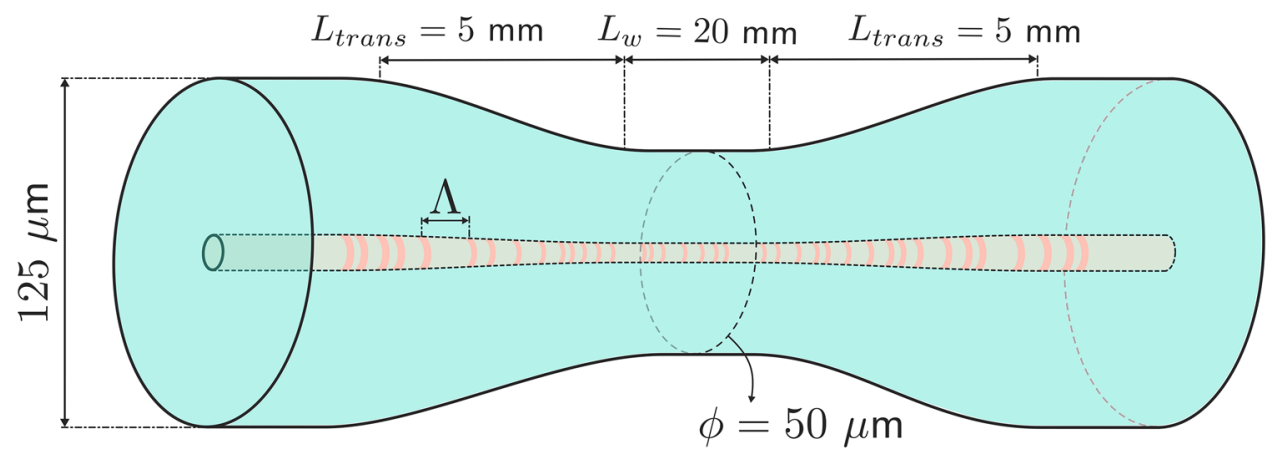

Figure 2. Schematic illustration of the $50 \mu \mathrm{m}$-waist tapered SMF, in which micro-modifications were applied using the femtosecond laser inscription setup.

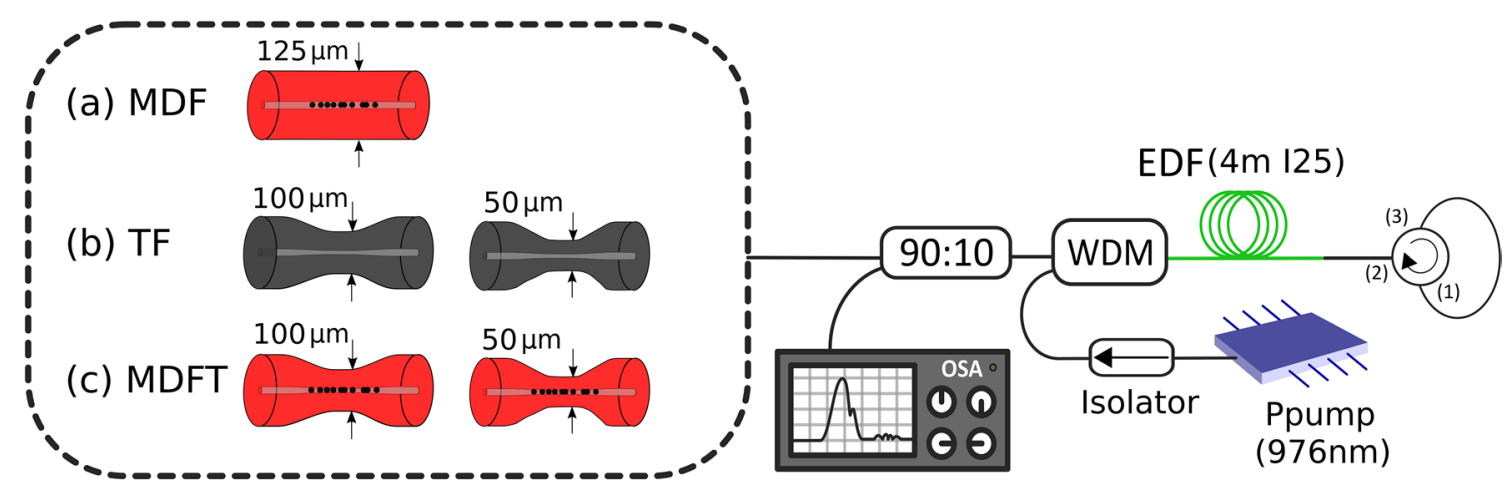

Figure 3. Schematic diagram of the experimental linear short-cavity fiber laser setup, in which (a) a microdrilled optical fiber (MDF), (b) un-drilled tapered optical fibers (TF) and (c) micro-drilled tapered fibers (MDFT) were used to reflect an amplified signal.

composition optimized for erbium-doped fiber amplifiers (EDFAs) in dense wavelength-division multiplexing (DWDM) networks. The peak core absorption ranges from 7.7 to $9.4 \mathrm{~dB} / \mathrm{m}$ at $1531 \mathrm{~nm}$. The linear cavity of the laser ends at a broadband reflector that consists of a fiber loop mirror (FLM) formed by an optical circulator in which ports 3 and 1 are connected ${ }^{9}$. After this, the recirculating signal travels through the 1550 -nm port of the WDM to an optical coupler. At the optical coupler (OC), $10 \%$ of the signal was monitored by an optical spectrum analyzer (OSA) with a resolution of $0.03 \mathrm{~nm}$, and the other $90 \%$ was guided to the distributed reflectors. All the experimental measurements were carried out at room temperature, and no vibration isolation or temperature compensation techniques were employed.

\section{Results and discussion}

Laser generation properties. It has been previously demonstrated by the authors that micro-drilling techniques have an enhancing effect not only on the laser generation ${ }^{9}$ but also on the sensor properties ${ }^{15}$. This is evidenced by the results presented $\mathrm{in}^{15}$, where some of the lasing and sensor properties when using a singlemode micro-drilled optical fiber (MDF) as a reflector were experimentally demonstrated.

Figure 4 shows the output spectra of a short-linear-cavity fiber laser using a single-mode MDF as a reflector. In that case, a single-wavelength laser centered at $1568.6 \mathrm{~nm}$ with an optical signal to noise (OSNR) level of $45 \mathrm{~dB}$ and an output power level of $-9.6 \mathrm{dBm}$ were obtained when pumped at $140 \mathrm{~mW}$. In addition to this, this linear short-cavity fiber laser presented an efficiency of about $0.08 \%$. Previous studies demonstrate that these values are reasonably good for utmost sensor applications ${ }^{16}$.

Then, the laser generation properties when using optical tapered fibers as reflectors, both un-drilled (TF) and micro-drilled (MDTF) are presented. Previous experimental studies presented the analysis of the backscattering generation in a $50 \mu \mathrm{m}$-waist diameter micro-drilled tapered fiber within a similar linear-cavity fiber laser ${ }^{9}$. In this case, the aim is to analyze in depth the impact of a $100 \mu \mathrm{m}$-waist and to evaluate the effect of using different pump power wavelengths.

Figures 5a,b present the reflection spectra of the $50 \mu \mathrm{m}$-waist and $100 \mu \mathrm{m}$-waist diameter micro-drilled tapered fiber, in that order. These reflection spectra show a fairly flat response, so the emission wavelength of the generated lasers will be determined mainly by the spectrum of the generated amplified spontaneous emission (ASE). For both cases, when the pumping wavelengths are centered at $1445 \mathrm{~nm}$ and $1480 \mathrm{~nm}$, the maximum output power level is around $1562 \mathrm{~nm}$.

Figure 6 shows a comparison between the results measured when using a $50 \mu \mathrm{m}$-waist or $100 \mu \mathrm{m}$-waist tapered fiber, as presented in Fig. 6a,b in that order. Each of these figures shows the output spectra for an 


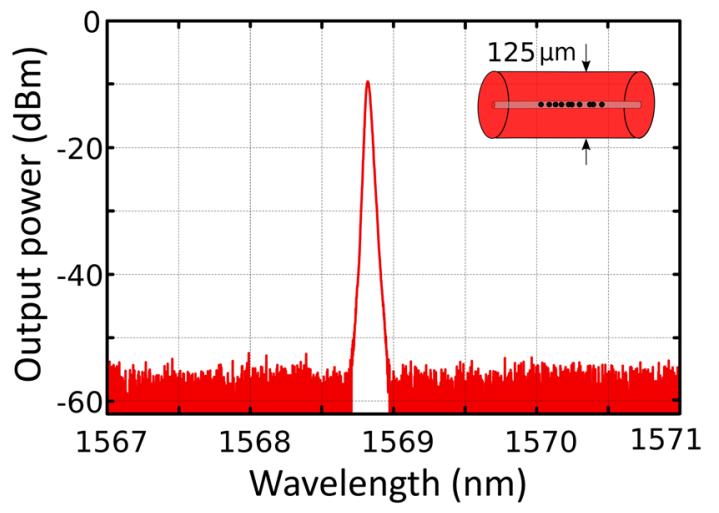

Figure 4. Output spectra of a short-linear-cavity fiber laser when using a single mode MDF pumped by a 976$\mathrm{nm}$ laser at $140 \mathrm{~mW}$.
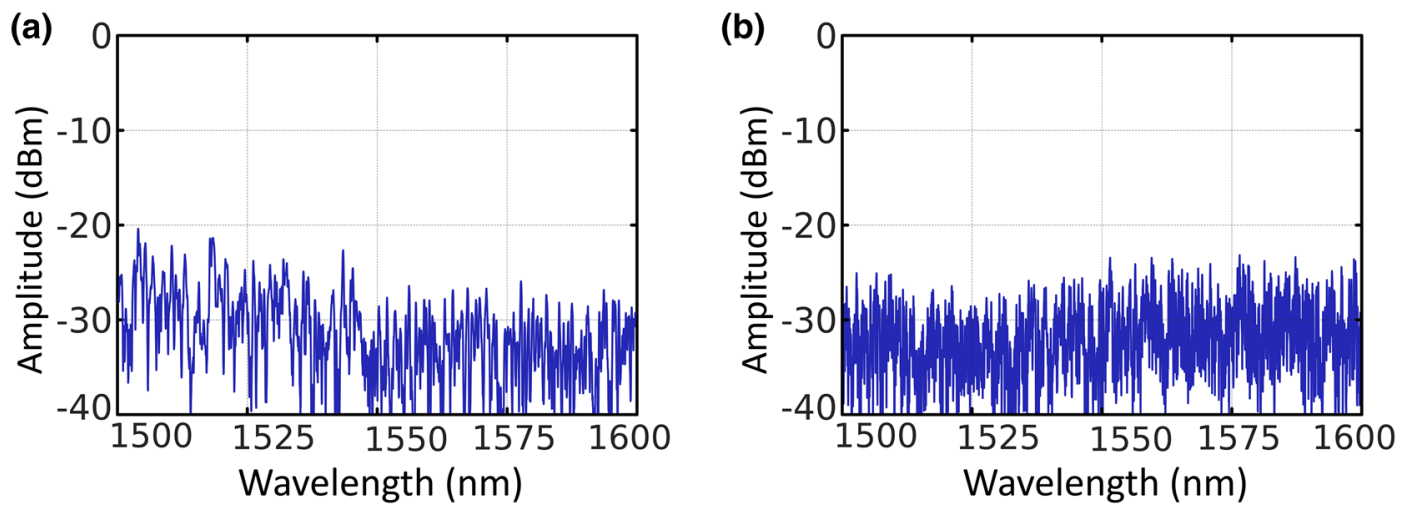

Figure 5. (a) Reflection spectrum of the $50 \mu \mathrm{m}$-waist micro-drilled tapered fiber. (b) Reflection spectrum of the $100 \mu \mathrm{m}$-waist micro-drilled tapered fiber.
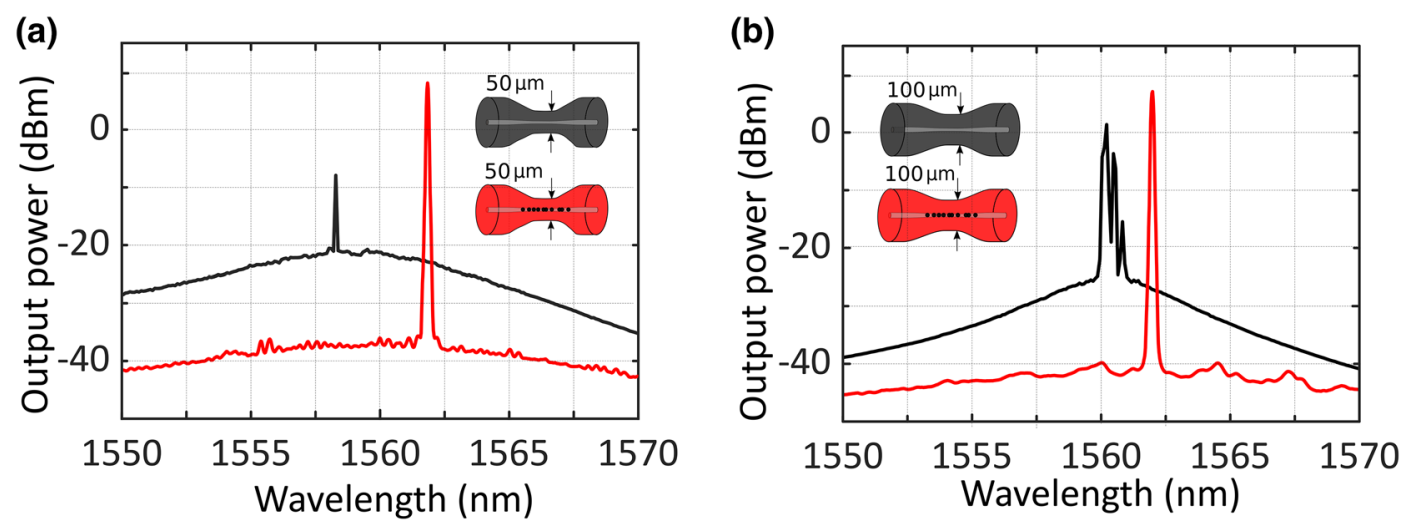

Figure 6. (a) Output spectra of the short-linear-cavity fiber laser, pumped by a 976-nm light at 350mW, when using a $50 \mu \mathrm{m}$-waist un-drilled (black line) or micro-drilled tapered fiber (red line). (b) Output spectra of the short-linear-cavity fiber laser, pumped by a $976-\mathrm{nm}$ light at $350 \mathrm{~mW}$ when using a $100 \mu \mathrm{m}$-waist un-drilled (black line) or micro-drilled tapered fiber (red line).

un-drilled tapered fiber (black line) or a micro-drilled tapered fiber (red line), both of them being pumped with $350 \mathrm{~mW}$ at 976-nm. As it was previously pointed out, the micro-drilling, even with a higher waist diameter and using a different pump laser, dramatically enhances the laser generation properties, as expected, due to the increase of the total reflectivity.

As Fig. 6a shows, when a $50 \mu \mathrm{m}$-waist un-drilled tapered fiber was used as reflector (black line), the smallsignal gain was not sufficient for single-wavelength lasing. However, when this reflector is replaced by a microdrilled tapered one, a single-wavelength laser centered at $1561.8 \mathrm{~nm}$ was emitted. The output power level obtained 
(a)

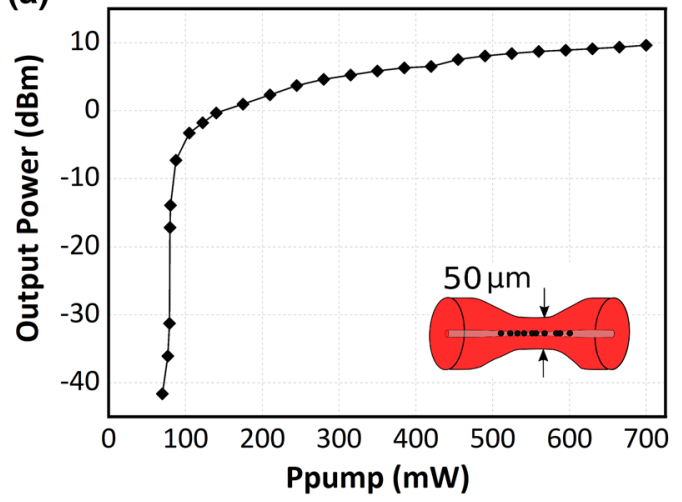

(b)

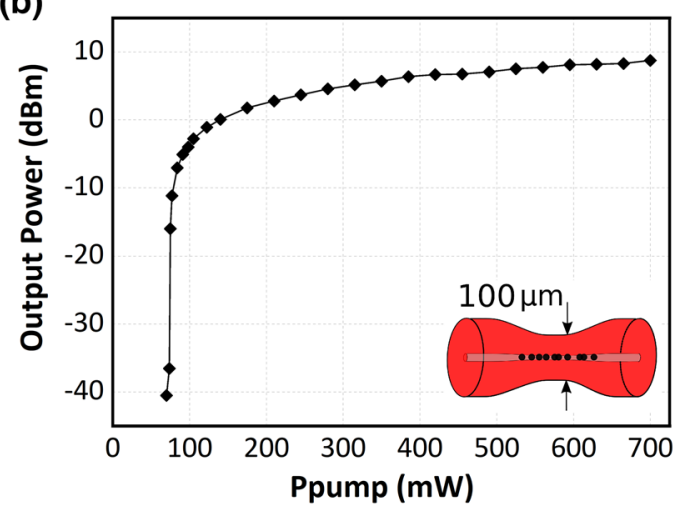

Figure 7. (a) Relationship between the output-power values as a function of 976-nm pump power for the $50 \mu \mathrm{m}$-waist MDTF-based fiber laser. (b) Relationship between the output-power values as a function of $976-\mathrm{nm}$ pump power for the $100 \mu \mathrm{m}$-waist MDTF-based fiber laser.

(a)

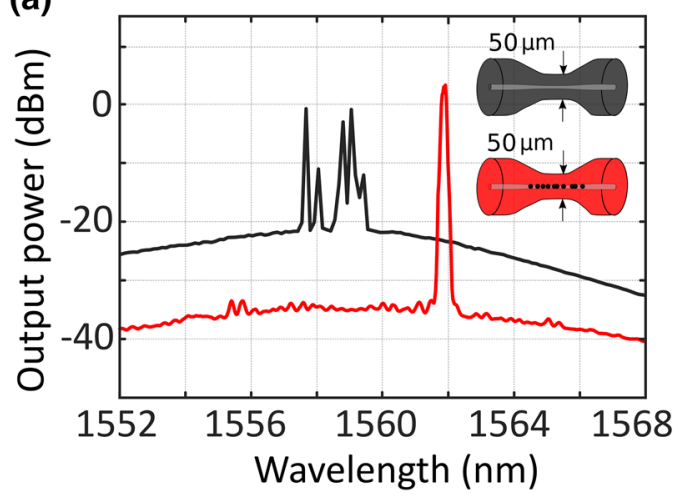

(b)

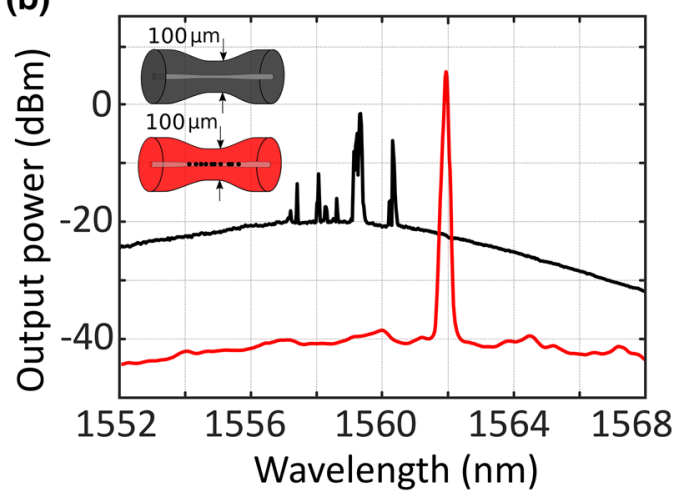

Figure 8. (a) Output spectra of the short-linear-cavity fiber laser, pumped by a 1445-nm light at $500 \mathrm{~mW}$ and using an un-drilled (black line) or a micro-drilled (red line) $50 \mu \mathrm{m}$-waist tapered fiber. (b) Output spectra of the short-linear-cavity fiber laser, pumped by a $1445-\mathrm{nm}$ light at $500 \mathrm{~mW}$ and using an un-drilled (black line) or a micro-drilled (red line) $100 \mu \mathrm{m}$-waist tapered fiber.

from this single-laser oscillation when pumped at $350 \mathrm{~mW}$ was $8.2 \mathrm{dBm}$, with an OSNR of $45 \mathrm{~dB}$ (red line). The efficiency of this linear short-cavity fiber laser was about $1.9 \%$.

This experimental comparison was also carried out by using $100 \mu \mathrm{m}$-waist tapers, as illustrated in Fig. 6b. In this case, when an un-drilled tapered was evaluated, the obtained output spectrum showed that, one more time, the small-signal gain was not sufficient for single-wavelength lasing and longitudinal-mode competition was observed (black line). On the other hand, when a $100 \mu \mathrm{m}$-waist micro drilled taper was used, a single-wavelength laser centered at $1562 \mathrm{~nm}$, with an output power level of $7.2 \mathrm{dBm}$ and an OSNR of $48 \mathrm{~dB}$ was measured (red line). In this case, the efficiency, evaluated under the same circumstances, was $1.5 \%$.

Figure 7a,b display the experimental results of the measured output power level as a function of the inserted 976-nm pump power for the $50 \mu \mathrm{m}$-waist and $100 \mu \mathrm{m}$-waist tapered fibers respectively, both presenting a similar pump power threshold of about $80 \mathrm{~mW}$.

As it is well known, erbium-doped fibers can be pumped not only by using wavelengths at the $980 \mathrm{~nm}$-band but also around $1480 \mathrm{~nm}$. To further study the properties of this short-linear-cavity laser when pumping at other central wavelengths, the pump laser centered at $976 \mathrm{~nm}$ was replaced by others centered at $1445 \mathrm{~nm}$ and $1480 \mathrm{~nm}$. Then, a similar experimental study was carried out for these four reflectors.

Figure 8 shows a comparison between the results attained using a $50 \mu \mathrm{m}$-waist or $100 \mu \mathrm{m}$-waist tapered fiber, as illustrated in Fig. 8a,b respectively, both of them being pumped with 500mW at 1445 -nm light. Once more, the micro-drilling, even with a higher waist diameter and using a different pump laser, dramatically enhances the laser generation properties.

When using an un-drilled reflector, both output spectra of $50 \mu \mathrm{m}$ and $100 \mu \mathrm{m}$ waist tapered fibers showed that a small-signal gain, not enough for single-wavelength lasing and longitudinal-mode competition, was observed (see black lines in Figs. 8). Nevertheless, when micro-drilled tapered fibers were used, a single laser emission line can be observed. In the case of using a $50 \mu \mathrm{m}$-waist tapered fiber, this single laser emission line was centered at $1561.9 \mathrm{~nm}$, with an output power level of $3.4 \mathrm{dBm}$ and an OSNR of $39 \mathrm{~dB}$. On the other hand, when a $100 \mu \mathrm{m}$-waist tapered fiber was used as reflector, this single laser emission line was also centered at $1561.9 \mathrm{~nm}$. However, an output power level of $5.6 \mathrm{dBm}$ and an OSNR of $45 \mathrm{~dB}$ were measured. 
(a)

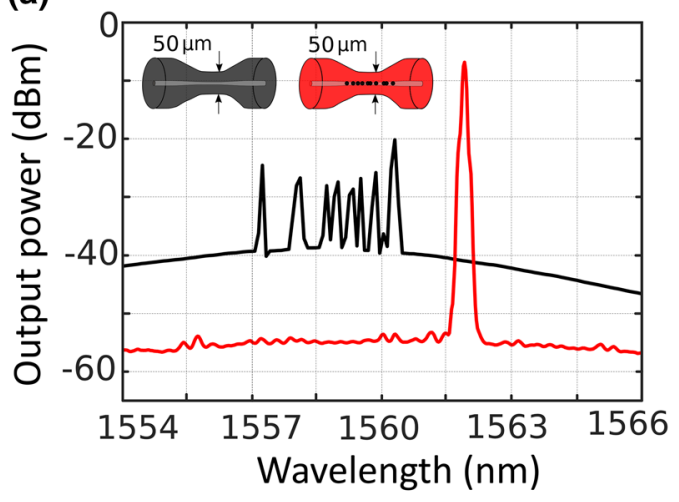

(b)

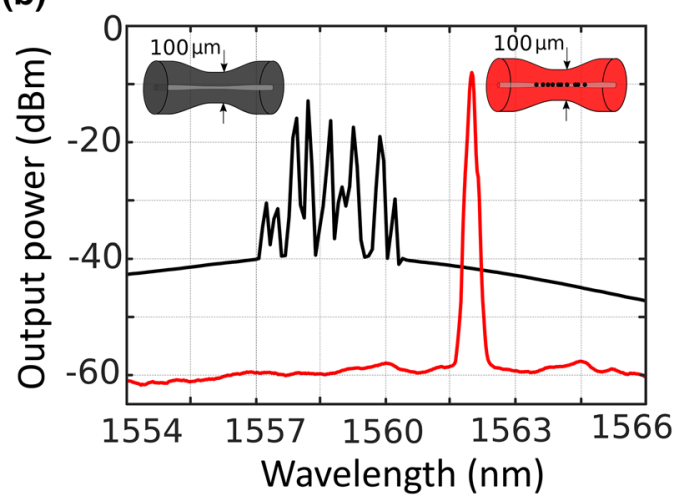

Figure 9. (a) Output spectra of the short-linear-cavity fiber laser, pumped by a 1480-nm light at $500 \mathrm{~mW}$ and using an un-drilled (black line) or a micro-drilled (red line) $50 \mu \mathrm{m}$-waist tapered fiber. (b) Output spectra of the short-linear-cavity fiber laser, pumped by a 1480-nm light at $500 \mathrm{~mW}$ and using an un-drilled (black line) or a micro-drilled (red line) $100 \mu \mathrm{m}$-waist tapered fiber.
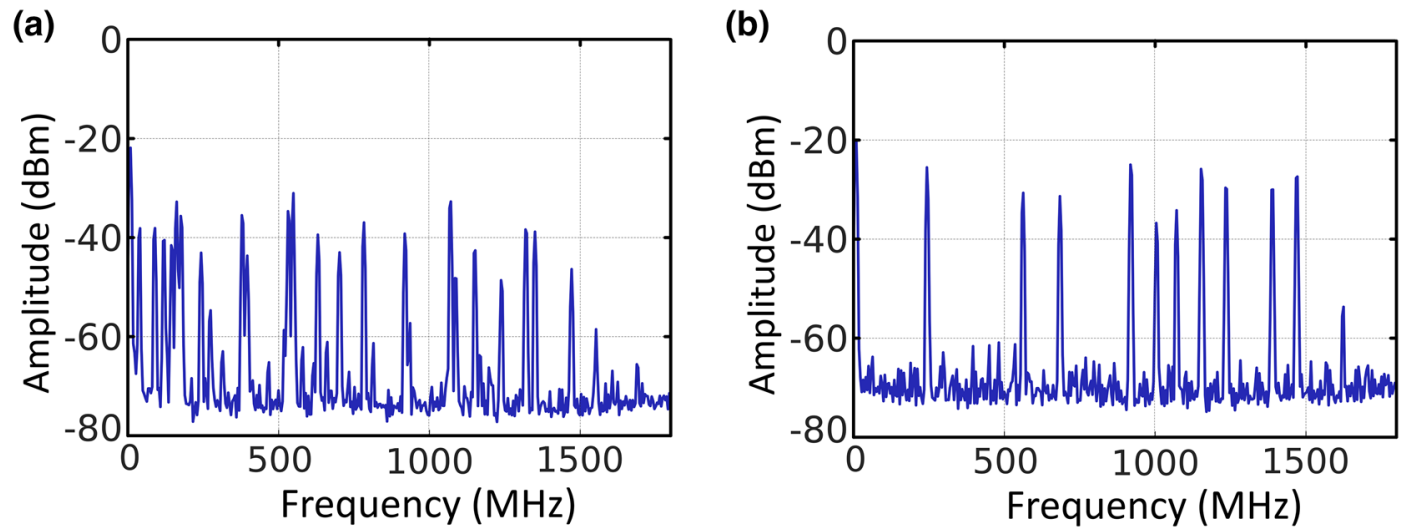

Figure 10. (a) Radio-frequency spectra of the beat of the $50 \mu \mathrm{m}$-waist tapered fiber with the TLS when pumped at 1480-nm light. (b) Radio-frequency spectra of the beat of the $100 \mu \mathrm{m}$-waist tapered fiber with the TLS when pumped at 1480 -nm light.

The output spectra of the short-linear-cavity fiber laser, when pumped by a $1480-\mathrm{nm}$ light at $500 \mathrm{~mW}$ is shown in Fig. 9. As expected, and despite using a different pumping wavelength, the micro-drilling significantly enhances the laser generation properties, as seen in the red lines in both Fig. 9a,b.

When a $50 \mu \mathrm{m}$-waist tapered fiber was used as a reflector, a single-wavelength laser centered at $1562 \mathrm{~nm}$, with an output power of $-6.9 \mathrm{dBm}$ and an OSNR of $48 \mathrm{~dB}$ (Fig. 9a red line). In this case, the optical efficiency of this linear short-cavity fiber laser was as low as $0.04 \%$. On the other hand, a single-wavelength laser also centered at $1562 \mathrm{~nm}$, with an output power of $-8 \mathrm{dBm}$, an OSNR of $51 \mathrm{~dB}$ and an optical efficiency of $0.03 \%$ were measured when a $100 \mu \mathrm{m}$-waist tapered fiber was used as a reflector (Fig. $9 \mathrm{~b}$ red line). It should be noted that, in this case, although the output power level has been reduced, the noise level has been further reduced, so the OSNR level is increased.

In order to experimentally characterize the longitudinal-mode behavior of these lasers, the output port, previously monitored by an OSA, was connected to a photodetector in combination with an electrical spectrum analyzer (ESA) to perform measurements in the electrical frequency domain. The reflected signal from the MDTFs were mixed with the signal form a tunable laser source (TLS, Agilent 8164B), though a $3 \mathrm{~dB}$ coupler to perform a heterodyne detection. Figures $10 \mathrm{a}, \mathrm{b}$ illustrate the frequency spectra corresponding to the frequency domain conversion, when using a $50 \mu \mathrm{m}$-waist and $100 \mu \mathrm{m}$-waist tapered fiber, respectively, both pumped by a 1480 -nm light. These two figures clearly show the appearance of multiple longitudinal mode beating so, presenting a multimode operation. Similar results were obtained when pumped by $976-\mathrm{nm}$ and 1445 -nm light.

It has been experimentally demonstrated that the obtained output spectra when using an un-drilled tapered fiber (TF) presents a small-signal gain because of its low reflectivity. However, it is not sufficient enough for single-wavelength lasing and, in addition to this, longitudinal mode competition was observed for all the different pumping wavelengths used in this work. For this reason, this TF reflector will not be analyzed in the following studies. 


\begin{tabular}{|l|l|l|l|l|l|}
\hline Optical fiber structure reflector & Pump power wavelength & Central wavelength & Output power & OSNR & Efficiency (\%) \\
\hline MDF & $976 \mathrm{~nm}$ & $1568.6 \mathrm{~nm}$ & $-9.6 \mathrm{dBm}$ & $45 \mathrm{~dB}$ & 0.08 \\
\hline \multirow{3}{*}{$50 \mu \mathrm{m}$-waist MDTF } & $976 \mathrm{~nm}$ & $1561.8 \mathrm{~nm}$ & $+8.2 \mathrm{dBm}$ & $45 \mathrm{~dB}$ & 1.89 \\
\cline { 2 - 7 } & $1445 \mathrm{~nm}$ & $1562 \mathrm{~nm}$ & $+3.4 \mathrm{dBm}$ & $38 \mathrm{~dB}$ & 0.44 \\
\cline { 2 - 7 } & $1480 \mathrm{~nm}$ & $1562 \mathrm{~nm}$ & $-6.9 \mathrm{dBm}$ & $48 \mathrm{~dB}$ & 0.04 \\
\hline \multirow{3}{*}{$100 \mu \mathrm{m}$-waist MDTF } & $976 \mathrm{~nm}$ & $1562 \mathrm{~nm}$ & $+7.2 \mathrm{dBm}$ & $48 \mathrm{~dB}$ & 1.5 \\
\cline { 2 - 7 } & $1445 \mathrm{~nm}$ & $1561.9 \mathrm{~nm}$ & $+5.8 \mathrm{dBm}$ & $45 \mathrm{~dB}$ & 0.76 \\
\cline { 2 - 6 } & $1480 \mathrm{~nm}$ & $1562 \mathrm{~nm}$ & $-8 \mathrm{dBm}$ & $51 \mathrm{~dB}$ & 0.03 \\
\hline
\end{tabular}

Table 1. Comparison of laser generation properties when using a micro-drilled fiber (MDF), a $50 \mu \mathrm{m}$-waist or a $100 \mu \mathrm{m}$-waist micro-drilled tapered as a reflector (MDTF).

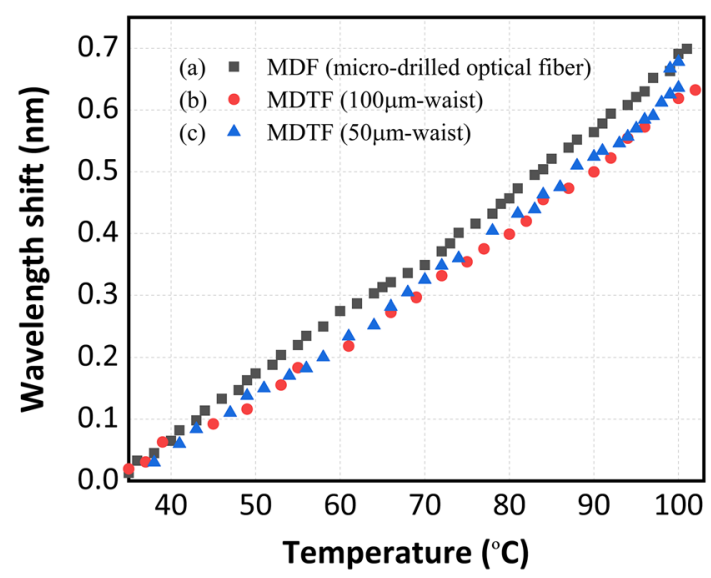

Figure 11. Wavelength shift as function of temperature when using (a) the MDF, (b) the MDTFs with $100 \mu \mathrm{m}$-waist or (c) the $50 \mu \mathrm{m}$-waist as temperature sensor.

Table 1 contains, in summary form, a comparison of the results obtained in the experiments. There, it is presented a comparison of the laser generation properties using a micro-drilled fiber, a 50 or a $100 \mu \mathrm{m}$-waist micro-drilled tapered fiber as a reflector, as a function of the inserted pump power wavelength.

Temperature and strain sensor. Considering the above results, both in terms of output power, OSNR level as well as optical efficiency, the properties of these reflectors as temperature and strain sensors were also analyzed. The following studies were carried out using a micro-drilled optical fiber (MDF), a $50 \mu \mathrm{m}$-waist MDTF and a $100 \mu \mathrm{m}$-waist MDTF all of them with a pump power wavelength of $976 \mathrm{~nm}$.

First, this structure was characterized as temperature sensor when no strain was applied. The wavelengthshift sensitivity to temperature was characterized using the MDF, the $50 \mu \mathrm{m}$-waist MDTF and the $100 \mu \mathrm{m}$-waist MDTF distributed reflectors. This characterization was carried out by using a climatic chamber in the range of $35^{\circ} \mathrm{C}$ to $100{ }^{\circ} \mathrm{C}$ and taking samples each $2{ }^{\circ} \mathrm{C}$.

As depicted in Fig. 11, the center wavelength-shift for these three single-wavelength lasers when using a MDF, a MDTF with $100 \mu \mathrm{m}$-waist or a MDTF with $50 \mu \mathrm{m}$-waist, (see Fig. 11a,b,c, respectively) present a clear linear behavior (the mean square errors were equal to $0.9983,0.9953$ and 0.9976 respectively) and temperature sensitivities of $10 \mathrm{pm} /{ }^{\circ} \mathrm{C}, 9.1 \mathrm{pm} /{ }^{\circ} \mathrm{C}$ and $9.6 \mathrm{pm} /{ }^{\circ} \mathrm{C}$ were measured, in that order. The attained values were close to the typical value for temperature-induced Bragg wavelength shift in silica fibers operating at $1550 \mathrm{~nm}$, which is around $11 \mathrm{pm} /{ }^{\circ} \mathrm{C}^{5}$.

In the view of the above, these sensor heads based on micro-drilled optical fibers, were placed in a high precision single-axis motorized stage (MS) in order to evaluate the wavelength shift induced by axial-strain changes. The characterization of the sensor heads consisted of 29 steps of $3.15 \mu \varepsilon$ per step.

As it is well known strain, unlike temperature, is a process mainly driven by the mechanical deformation of the optical fiber when a longitudinal stretch is applied. The taper sensitivity to strain is mainly determined by the physical dimensions of the sensor system, as it has been previously studied and demonstrated ${ }^{17}$. This deformation is inversely proportional to the radius of the optical fiber, so the smaller the diameter, the greater the effect of the strain applied on the sensing part. The strain loads applied to a FBG and a tapered fiber are related according to:

$$
\Delta \varepsilon_{F B G} E A_{F B G}=\Delta \varepsilon_{\text {taper }} E A_{\text {taper }}
$$




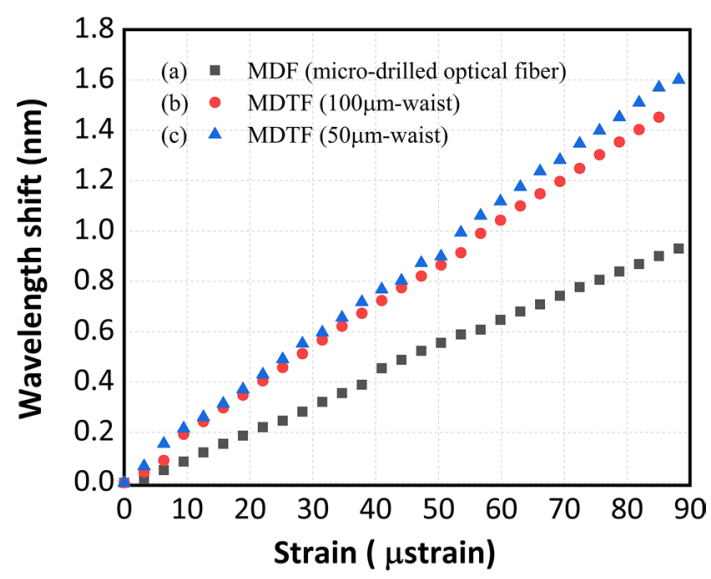

Figure 12. Wavelength shift as a function of strain change when using (a) a MDF, (b) a MDTF with $100 \mu \mathrm{m}$-waist, and (c) a MDTF with $50 \mu \mathrm{m}$-waist.

\begin{tabular}{|l|l|l|l|}
\hline Optical fiber structure & Waist diameter & Temperature sensitivity & Strain sensitivity \\
\hline $\mathrm{FBG}^{5}$ & $125 \mu \mathrm{m}$ & $11 \mathrm{pm} /{ }^{\circ} \mathrm{C}$ & $1.2 \mathrm{pm} / \mu$ strain \\
\hline $\mathrm{MDF}$ & $125 \mu \mathrm{m}$ & $10.5 \mathrm{pm} /{ }^{\circ} \mathrm{C}$ & $10.9 \mathrm{pm} / \mu$ strain \\
\hline $\mathrm{MDTF}$ & $100 \mu \mathrm{m}$ & $9 \mathrm{pm} /{ }^{\circ} \mathrm{C}$ & $17 \mathrm{pm} / \mu$ strain \\
\hline $\mathrm{MDTF}$ & $50 \mu \mathrm{m}$ & $9.5 \mathrm{pm} /{ }^{\circ} \mathrm{C}$ & $18.1 \mathrm{pm} / \mu$ strain \\
\hline
\end{tabular}

Table 2. Comparison of temperature and strain sensitivity for a FBG, micro-drilled fiber or micro-drilled tapered fiber.

where $\boldsymbol{E}$ is the Young modulus of the sensor material, $A_{F B G}$ and $A_{\text {taper }}$ are the cross-sectional areas in the FBG and in the tapered optical fiber, respectively, and $\varepsilon_{F B G}$ and $\varepsilon_{\text {taper }}$ are the strain applied on the FBG or the tapered fiber, in that order ${ }^{17}$. Due to the refractive index modifications caused by the inscription process, the mechanical and optical properties of the material in a FBG or the tapered optical fibers are not the same. So, the strain applied to these reflectors will depend not only on the ratio of the cladding diameters but also to the mechanical properties of each one of them. Consequently, in a complex structure such as these micro-drilled tapered fibers (MDTFs), not only the dimensions of the tapered fiber waist affect, but also the transitions between the original optical fiber and the uniform waist of the taper or the refractive index modifications among others, also modify the response of the sensor.

In order to quantify the effect only of those refractive index modifications and not the fiber core dimensions, the first experiment carried out was with a micro-drilled standard single-mode optical fiber, with a diameter of 125 microns, in order to compare its response with that of a FBG, also with the same diameter, and to see if these inscriptions provided any improvement in terms of the sensor's sensitivity. After analyzing this first structure with a size similar to that of a FBG, a more detailed study was carried out for reflectors with smaller waist diameters, from $125 \mu \mathrm{m}$ to $100 \mu \mathrm{m}$ and $50 \mu \mathrm{m}$.

Figure 12 presents the central emission wavelength shift when the structure was subjected to the abovementioned strain variations using (a) a MDF, (b) a MDTF with $100 \mu \mathrm{m}$-waist, and (c) a MDTF with $50 \mu \mathrm{m}$-waist. These results show linear response as evidenced by the R-squared values, close to $1(0.9983,0.9991$ and 0.9992 respectively), showing sensitivities as good as $10.9 \mathrm{pm} / \mu \varepsilon, 17 \mathrm{pm} / \mu \varepsilon$ and $18.1 \mathrm{pm} / \mu \varepsilon$ for the MDF, the MDTFs with $100 \mu \mathrm{m}$-waist or $50 \mu \mathrm{m}$-waist, in that order.

These values, when compared with the typical value for strain-induced Bragg wavelength shift that is approximately $1.2 \mathrm{pm} / \mu \varepsilon^{5,6}$, present a significant enhancement of more than one order of magnitude. This represents a substantial improvement of the strain sensitivity.

Finally, Table 2 provides a summary of the data acquired over this experimental study. This table shows a temperature and strain comparison for the sensor heads of interest as well as the typical values for temperature and strain induced Bragg sensor. Although temperature sensitivities achieved were similar to FBGs, the strain sensitivity presents more than one order of magnitude enhancement.

\section{Conclusions}

In conclusion, this work presents an experimental performance analysis of different types of quasi-randomly distributed reflectors written into single-mode fiber, when used as sensing mirrors or sensor heads in a shortlinear-cavity fiber laser. In particular, the features of the laser generation when optical tapered fibers (TF), microdrilled optical fibers (MDF) or two different micro-drilled tapered fibers (MDTF) are presented. Regarding the 
laser generation properties, the best results in terms of output power level, OSNR, or optical efficiency, were obtained for the case of micro-drilled tapered optical fiber when pumped at $976 \mathrm{~nm}$. In particular, when the $50 \mu \mathrm{m}$-waist or $100 \mu \mathrm{m}$-waist micro-drilled tapered optical fibers were used, a single-wavelength reflector laser centered at $1561.8 \mathrm{~nm}$ or $1562 \mathrm{~nm}$, with an output power level of $8.2 \mathrm{~dB}$ or $7.2 \mathrm{~dB}$ and an OSNR higher than $45 \mathrm{~dB}$ or $48 \mathrm{~dB}$ were measured respectively.

In terms of the properties as a sensor, temperature sensitivities of around $10 \mathrm{pm} /{ }^{\circ} \mathrm{C}$ were measured. However, and even though the achieved temperature sensitivities were close to the typical value for temperature-induced Bragg wavelength shift in silica fibers operating at $1550 \mathrm{~nm}$, the strain sensitivity was significantly improved. As an example, when the $50 \mu \mathrm{m}$-waist MDTF was employed as distributed reflector a strain sensitivity as high as $18.1 \mathrm{pm} / \mu \varepsilon$ was measured, which means an improvement of more than one order of magnitude in comparison to FBG sensors.

Received: 29 July 2021; Accepted: 1 October 2021

Published online: 14 October 2021

\section{References}

1. Cusano, A., Cutolo, A. \& Albert, J. Fiber Bragg grating sensors: Recent advancements, industrial applications and market exploitation. Bentham Science Publishers https://doi.org/10.2174/97816080508401110101 (2013).

2. Annamdas, V.G.M., Yang Y., \& Liu, H. Current development in fiber Bragg grating sensors and their applications, Proc. SPIESensors and Smart Structures Technologies for Civil, Mechanical, and Aerospace Systems, 6932 (2008). https://doi.org/10.1117/ 12.775715

3. Kersey, A. D. \& Morey, W. W. Multi-element Bragg-grating based fibre-laser strain sensor. Electron. Lett. 29(11), 964-966. https:// doi.org/10.1049/el:19930642 (1993).

4. Avino, S., Giorgini, A. \& Gagliardi, G. Fiber-optic cavities for physical and chemical sensing. Open Opt. J. 7, 128-140. https://doi. org/10.2174/1874328501307010128 (2013).

5. Grattan, L.S., \& Meggit, B.T. Optical Fiber Sensor Technology: Devices and Technology. Vol. 2 (Optoelectronics, Imaging and Sensing, Springer Science \& Business Media, 2012). DOI: https://doi.org/10.1007/978-1-4615-5787-6

6. Campanella, C. E., Cuccovillo, A., Campanella, C., Yurt, A. \& Passaro, V. M. N. Fibre Bragg grating based strain sensors: Review of technology and applications. Sensors 18(9), 3115. https://doi.org/10.3390/s18093115 (2018).

7. Malo, B., Hill, K. O., Bilodeau, F., Johnson, D. C. \& Albert, J. Point-by-point fabrication of micro-Bragg gratings in photosensitive fibre using single excimer pulse refractive index modification techniques. Electron. Lett. 29(18), 1668-1669. https://doi.org/10. 1049/el:19931110 (1993).

8. Wang, X., She, L., Chen, D. \& Wu, Q. Theoretical and experimental study of artificially controlled backscattering fiber using femtosecond laser fabrication. Opt. Laser Technol. 113, 6-10. https://doi.org/10.1016/j.optlastec.2018.11.052 (2019).

9. Perez-Herrera, R. A. et al. Optical fiber lasers assisted by microdrilled optical fiber tapers. Opt. Lett. 44, 2669-2672. https://doi. org/10.1364/OL.44.002669 (2019).

10. Perez-Herrera, R. A. et al. Hybrid Raman-erbium random fiber laser with a half open cavity assisted by artificially controlled backscattering fiber reflectors. Sci. Rep. 11, 9169. https://doi.org/10.1038/s41598-021-88748-w (2021).

11. Perez-Herrera, R. A., Roldán-Varona, P., Rodriguez-Cobo, L., Lopez-Higuera, J. M. \& Lopez-Amo, M. Single longitudinal mode lasers by using artificially controlled backscattering erbium doped fibers. IEEE Access 9, 27428-27433. https://doi.org/10.1109/ ACCESS.2021.3058092 (2021).

12. Gattass, R. \& Mazur, E. Femtosecond laser micromachining in transparent materials. Nat. Photon. 2, 219-225. https://doi.org/10. 1038/nphoton.2008.47 (2008).

13. Zhou, K., Shen, F., Yin, G., \& Zhang, L. Optical fiber micro-devices made with femtosecond laser. In Advanced Photonics 2016 (IPR, NOMA, Sensors, Networks, SPPCom, SOF), OSA Technical Digest (online) (Optical Society of America, 2016), paper SeW3D.1. https://doi.org/10.1364/SENSORS.2016.SeW3D.1

14. Judez, A., Leandro, D., Perez-Herrera, R. A., Galarza, M., \& Lopez-Amo, M. High sensitivity fiber-optic liquid level sensor using biconical tapered fibers. 26th International Conference on Optical Fiber Sensors, OSA Technical Digest (Optical Society of America, 2018), paper TuE45. https://doi.org/10.1364/OFS.2018.TuE45

15. Perez Herrera, R. A., et al. Micro-drilled optical fiber for enhanced laser strain sensors. Proc. SPIE 11199, Seventh European Workshop on Optical Fibre Sensors, 111992R (2019). https://doi.org/10.1117/12.2539802

16. Pevec, S. \& Donlagić, D. Multiparameter fiber-optic sensors: a review. Opt. Eng. 58(7), 072009. https://doi.org/10.1117/1.OE.58.7. 072009 (2019).

17. Frazão, O. et al. Strain sensitivity control of fiber Bragg grating structures with fused tapers. Appl. Opt. 46(36), 8578-8582. https:// doi.org/10.1364/AO.46.008578 (2007).

\section{Acknowledgements}

This work is part of the project PID2019-107270RB, funded by MCIN/ AEI/10.13039/501100011033 and FEDER "A way to make Europe", the Ministerio de Educación, Cultura y Deporte of Spain (PhD grant FPU2018/02797), the European Union's Horizon 2020 research and innovation programme under the Marie Skłodowska-Curie grant agreement No 838143, the Beatriz Galindo BEAGAL18/00116 fellowship, the "Fundación Caja Navarra" and "Fundación la Caixa" with the proyect LCF/PR/PR13/51080004 and Projects for young researches UPNA 2019.

\section{Author contributions}

R.A.P.-H., M.B., P.R.-V. and D.L. designed and implemented the random fiber laser structure. P.R.-V. made the laser inscriptions for the fiber reflectors. R.A.P.-H and P.R.-V. prepared figures. R.A.P.-H., P. R-V. and M. L.-A. wrote the main manuscript text. L.R.-C., J.M. L.-H. and M.L-A. supervised the work.

\section{Competing interests}

The authors declare no competing interests.

Additional information

Correspondence and requests for materials should be addressed to R.A.P.-H. 
Reprints and permissions information is available at www.nature.com/reprints.

Publisher's note Springer Nature remains neutral with regard to jurisdictional claims in published maps and institutional affiliations.

(c) (i) Open Access This article is licensed under a Creative Commons Attribution 4.0 International License, which permits use, sharing, adaptation, distribution and reproduction in any medium or format, as long as you give appropriate credit to the original author(s) and the source, provide a link to the Creative Commons licence, and indicate if changes were made. The images or other third party material in this article are included in the article's Creative Commons licence, unless indicated otherwise in a credit line to the material. If material is not included in the article's Creative Commons licence and your intended use is not permitted by statutory regulation or exceeds the permitted use, you will need to obtain permission directly from the copyright holder. To view a copy of this licence, visit http://creativecommons.org/licenses/by/4.0/.

(C) The Author(s) 2021 